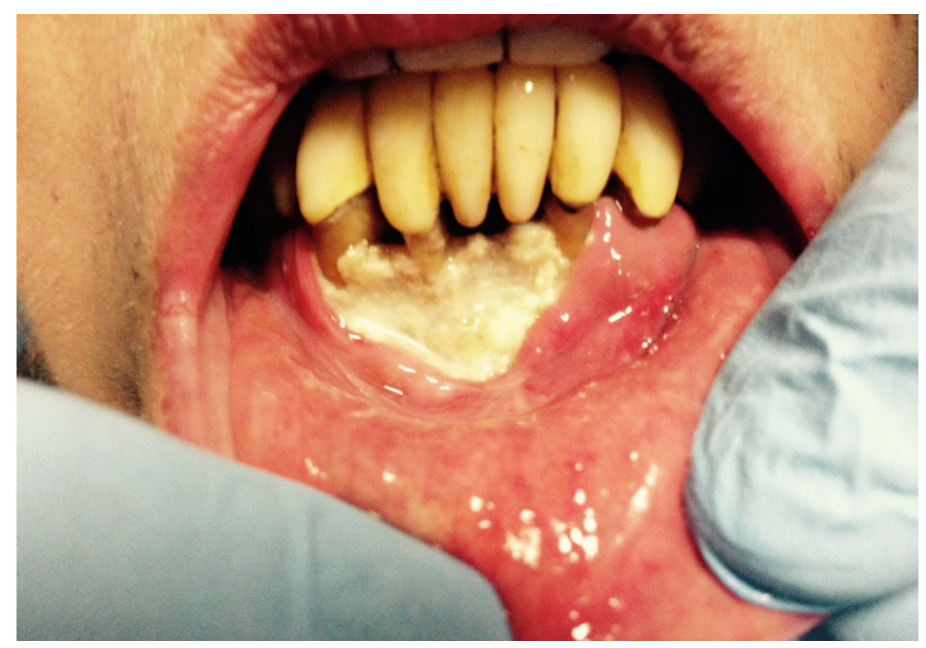

\title{
Prevention and screening of Osteonecrosis of Jaw (ONJ): the Alessandria experience of a multidisciplinary team on a 900-patient population
}

\author{
Antonella Fasciolo ${ }^{1}$, Vittorio Fusco ${ }^{1}$, Iolanda De Martino, Manuela Alessio ${ }^{2}$, Paola Brigo, Daniela Tartara ${ }^{1}$, Riccardo \\ Alberto Campora ${ }^{1}$, Maura Rossi ${ }^{1}$
}

1 Azienda Ospedaliera SS. Antonio e Biagio e Cesare Arrigo

2 Ospedale Alba-Bra

Funding: The author(s) received no specific funding for this work.

Potential competing interests: The author(s) declared that no potential competing interests exist.

\section{Abstract}

Patients at risk of Osteonecrosis of the Jaw (ONJ) include mostly cancer and myeloma patients receiving antiresorptive treatments (bisphosphonates and denosumab) with/without biological agents, but also patients with osteoporosis and rheumatologic disorders. ONJ cases were firstly diagnosed in our Hospital on 2005. Since 2006, a ONJ Multidisciplinary Team was established (including maxillofacial surgeons / dentists, oncologists, hematologists, nurses, radiologists, nuclear medicine and other medicine specialists, data managers). Preventive (risk reduction) measures before antiresorptive treatment (dental visit, dental panoramic Rx, eventual teeth extractions, dental and denture care) have been planned, according to early recommendations and their modifications. Furthermore in the Osteonecrosis Prevention and Screening office other patients received care if : a) ONJ was suspected after treatment for cancer and myeloma in neighboring hospitals, or b) ONJ was suspected among osteoporosis patients in the territory by private practice dentists. More than 900 patients were visited and registered in 2006-2020 years. Cases of suspected ONJ were 127 (45 male, 82 female). Twenty-four cases were excluded by analysis due to unconfirmed 
diagnosis or insufficient data. Characteristics of 103 cases of ONJ confirmed according to Italian (SIPMOSICMF) definition and recommendations were reviewed. Disease: 40 breast cancer; 16 prostate cancer; 11 myeloma; 7 renal cell cancer; 4 lung cancer; 7 other cancers; 18 osteoporosis and other non malignant disorders. Distribution of drugs associated to ONJ (zoledronic acid, denosumab, pamidronate, alendronate, risedronate, ibandronate, clodronate, bevacizumab, sunitinib) changed along years.

Background. Osteonecrosis of the Jaw (ONJ) is uncommon (not rare) in bone metastatic cancer and myeloma patients receiving antiresorptive treatments (bisphosphonates and denosumab) with/without biological agents, due to reduce Skeletal Related Events (SRE). ONJ is also diagnosed in patients receiving bisphosphonates and denosumab for osteoporosis and other non malignant bone disorders. Antiresorptive drugs include Bisphosphonates (BPs), such as pamidronate, zoledronic acid, ibandronate, etc, and an anti-RANKL agent, denosumab. All these agents are able to reduce the risk of SRE and to delay SRE onset in bone metastatic cancer and myeloma patients, and to reduce fracture risk in osteoporosis patients. However they are associated with adverse events, including Osteonecrosis of Jaw (ONJ), that can occur in 1-15\% of cancer and myeloma patients within first years of observation, whereas the risk for osteoporosis patients is lower (even still exactly not well known). Preventive (risk reduction) measures before BPs and denosumab treatment (dental visit, dental panoramic RX, eventual teeth extractions, dental and denture care) have been recommended. Furthermore screening visits are recommended in patients undergoing those drugs along treatment (often continuing for years).

Since 2006, a ONJ Multidisciplinary Team was established in Alessandria hospital, Italy; it includes maxillofacial surgeons / dentists, oncologists, hematologists, nurses, radiologists, nuclear medicine and other medicine specialists, data

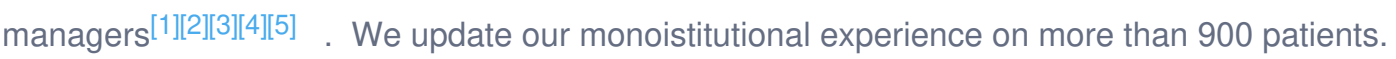

Materials and Methods: We reviewed charts of 905 patients followed by a multidisciplinary team at Alessandria Hospital in years 2006-2020. They were classified as follows :

1. Patients who had to start antiresorptive treatment, presenting bone metastases of solid tumors or myeloma, and followed by Oncology and Hematology Units of the Alessandria Hospital. They underwent dental visit, dental RX, eventual teeth extractions, dental and denture care.

2. Cancer and myeloma patients already treated with antiresorptive drugs, with dental problems or suspected ONJ, sent by colleagues of neighbour hospitals.

3. Patients with osteoporosis, Rheumatoid Arthirtis or other non-malignant bone diseases, suspected for ONJ, sent predominantly by private dental practitioners.

Cases were classified according to disease, drug administered, treatment duration, year of treatment start, and the dental history observed after start of antiresorptive treatments (bisphosphonates and/or denosumab).

Results: We followed 127 cases (45 male, 82 female) of suspected ONJ ("potential" ONJ cases) after treatment with BPs and/or denosumab and/or antiangiogenics drugs. After clinical and imaging evaluation, mostly by Computed Tomography 
(CT scan) and occasionally by nuclear medicine exams, ONJ was confirmed ("adjudicated" cases) in 103/127 patients

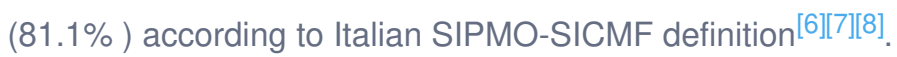

Patient disease: 40 (38.8\%) breast cancer ; 16 (15.5\%) prostate cancer; 11 (10.6\%) myeloma; 7 (6.8\%) renal cell cancer; 4 (3.9\%) lung cancer; 7 (6.8\%) other cancers; 18 (17.4\%) osteoporosis and other non malignant disorders.

The main drug involved in the ONJ development was: zoledronic acid in 44, denosumab (monthly $120 \mathrm{mg}$ ) in 10, denosumab (60 mg every 6 months) in 3, pamidronate in 9, ibandronate (several dosages and schedules ) in 5, alendronate in 6, antinagiogenics in 4 (2 bevacizumab, 2 sunitinib) , drug sequences or other bisphosphonates in 16 . Comparison of characteristics of ONJ patients with those not developing $\mathrm{ONJ}$ is ongoing; preliminary reports indicate that ONJ was associated to drug (higher risk for denosumab and zoledronic acid than for pamidronate), prolonged treatment (> 2 years), ill fitting dentures, periodontal disease.

Conclusions. : Medication-related $\mathrm{ONJ}$ is not rare. Further evaluations are planned to investigate the changes observed over latest fifteen years (of involved drugs; antiresorptive treatment duration; prolonged survival in different cancer types, etc), to obtain tools of prediction of ONJ risk for every single patient.

\section{References}

1. ' V. Fusco, A. Baraldi, A. Fasciolo, A. Pertino, and R. Gaino. (2014).Decreased occurrence of ONJ after preventive measures: the Alessandria experience on a 471 bisphosphonate patient population. Ann Stomatol (Roma). 2014 AprJun; 5(2 Suppl): 45-46..

2. `M. Vincenti, A. Fasciolo, P. Pepe, A. Pertino, and V. Fusco. (2014).ONJ (osteonecrosis of jaw) in breast cancer patients: effect of preventive measures in a mono institutional experience. Ann Stomatol (Roma). 2014 Apr-Jun; 5(2 Suppl): $14 .$.

3. ^P. Guglielmini, V. Fusco, A. Fasciolo, P. Pepe, and A. Pertino. (2014).Osteonecrosis of jaw (ONJ) in prostate cancer patients: report of a monoinstitutional experience. Ann Stomatol (Roma). 2014 Apr-Jun; 5(2 Suppl): $43 .$.

4. ' G. Catania, F. Monaco,A. Baraldi, A. Fasciolo, and A. Pertino. (2014).ONJ (osteonecrosis of jaw) in myeloma patients: a monoistitutional experience of 117 patients treated with bisphosphonates. Ann Stomatol (Roma). 2014 AprJun; 5(2 Suppl): 7-8..

5. `A. Pertino, R. Gaino, D. Tartara, and M. G. Candeo. (2014).Role of nurses in a multidisciplinary team for prevention, diagnosis, treatment and follow-up of osteonecrosis of jaw (ONJ). Ann Stomatol (Roma). 2014 Apr-Jun; 5(2 Suppl): 31-32..

6. ^A Bedogni, V Fusco, A Agrillo, G Campisi. (2012).Learning from experience. Proposal of a refined definition and staging system for bisphosphonate-related osteonecrosis of the jaw (BRONJ). Oral Dis . 2012 Sep;18(6):621-3. doi: 10.1111/j.1601-0825.2012.01903.x. Epub 2012 Feb 22..

7. ^Alberto Bedogni, Giuseppina Campisi, Vittorio Fusco, Alessandro Agrillo. (2013). Raccomandazioni clinicoterapeutiche sull'osteonecrosi delle ossa mascellari associata a bisfosfonati e sua prevenzione. Cleup.

8. 'Giuseppina Campisi, Alberto Bedogni, Vittorio Fusco. (2020). Raccomandazioni clinico-terapeutiche sull'osteonecrosi delle ossa mascellari (ONJ) farmaco-relata e sua prevenzione. Upapress. 
